\title{
Socially-Optimal Locations of Duopoly Firms with Non-Uniform Consumer Densities
}

\author{
Kieron J. Meagher ${ }^{1}$, Ernie G. S. Teo ${ }^{2}$, Taojun Xie ${ }^{3}$ \\ ${ }^{1}$ Research School of Economics, Australian National University, Canberra, Australia \\ ${ }^{2}$ Sim Kee Boon Institute of Financial Economics, Singapore Management University, Singapore \\ ${ }^{3}$ Division of Economics, Nanyang Technological University, Singapore \\ Email: kieron.meagher@anu.edu.au, ernieteo@smu.edu.sg, txie1@e.ntu.edu.sg
}

Received 11 April 2014; revised 11 May 2014; accepted 2 June 2014

Copyright (C) 2014 by authors and Scientific Research Publishing Inc.

This work is licensed under the Creative Commons Attribution International License (CC BY). http://creativecommons.org/licenses/by/4.0/

(c) ()

\begin{abstract}
Advances in the theoretical literature have extended the Hotelling model of spatial competition from a uniform distribution of consumers to the family of log-concave distributions. While a closed form has been found for the equilibrium locations for symmetric log-concave distributions, the literature contains no closed form solution for the socially optimal locations. We provide a closed form solution for the socially optimal locations: one mean-deviation away from the median. We also derive a formula for the excess differentiation ratio which complements the bounds previously derived in the literature, and establish the invariance of this ratio to a form of mean preserving spread. The equilibrium duopoly locations of several types of commonly used distributions were discussed in [1]. This paper provides the closed form solutions for the socially optimal locations to the same set of distributions. We calculate welfare improvements arising from regulation of firm location and show how these vary with the distribution of consumers. While regulating firm locations is sufficient to optimize welfare for symmetric distributions, additional price regulation is required to ensure social optimality for asymmetric distributions. These results are significant for urban policy over firm/store locations.
\end{abstract}

\section{Keywords}

Socially Optimal Firm Locations, Distributions, Spatial Competition, Duopoly

\section{Introduction}

Transport costs are a primary driver of the location decisions of firms in models of spatial economics. Transport costs can occur in an enormous range of contexts: inputs costs for intermediate products or in commute times for 
workers; or in output markets as search costs, shopping costs or delivery costs for consumers. A large, but by no means exhaustive, survey is provided by [2]. From a firm's perspective the essential issue of spatial economics is that it cannot be close to everything ${ }^{1}$. Identifying how firms handle this trade-off in choosing locations, and how these choices impact on society, gives rise to a central research theme of the literature, and it is to this area that this paper contributes.

Most theoretical firm location models assume a uniform consumer distribution; this is seldom observed in real life. In their seminal contributions [3] and [4] established the existence of price equilibrium for log-concave densities. [5] extended this line of research by providing sufficient conditions for the existence of pure strategy location-price equilibrium for log-concave consumer densities, and for the symmetric case, a closed form equation for the equilibrium locations and prices.

The analysis of socially optimal locations in the literature has not established a closed form for the socially optimal locations and as a result the comparison of equilibrium and optimal locations-in terms of the ratio of competitive product differentiation to the optimal differentiation-has produced bounds but not an exact result. We fill this gap by providing explicit formulas for both the socially optimal locations and the ratio of equilibrium to optimal differentiation.

The research questions of this paper are: 1) what are the socially optimal locations of firms within the canonical, quadratic Hotelling model; and 2) how do the equilibrium, firm level location decisions compare with the socially optimal locations? Our contribution is to extend the analysis of social optimality beyond the typical assumption of uniformly distributed consumers. In most real world settings, non-uniform consumer distributions would be more realistic — our results apply to important specific densities like the Normal and Logistic. One of the few theoretical works, which utilizes a non-uniform consumer distribution, is that of [6] where a symmetric triangular density was used. This is extended in unpublished work by [7], where the consumer distribution can take several forms ranging from uniform (rectangular) to triangular (densities in-between the two assume a trapezoidal shape). In [8], a state space approach where different states give rise to different consumer distributions was used to model uncertainty in spatial duopolies.

[1] identified a list of distributions where unique pure strategy equilibrium exists and also determines their locations. This paper follows [1] and examines the socially optimal locations of these distributions. Although [9] showed that there is excessive differentiation in the uniform case of the two-stage location-price game, and [5] supports the existence of excessive differentiation by deriving bounds results about the social-optimum locations; we are the first to examine precisely excess differentiation for a wider range of specific distributions, including asymmetric distributions.

The amount of differentiation and the total transport cost ${ }^{2}$ savings from government intervention are discussed in this paper. We also consider the effect of regulating the firms to the social-optimum locations and allowing them to set competitive prices. This decomposes the welfare gains from regulation into spatial and pricing decisions. This separation is important in practice because location in many industries is much cheaper to regulate/monitor than prices.

Using location as a form of regulation is of interest to governments. For example, the Australian Competition and Consumer Commission found in an inquiry into grocery stores in Australia that incumbent supermarkets have used planning objection processes to deter new entry thus lessening competition. Proceedings in the UK Competition Commission inquiry into Groceries [12] resulted in legal orders regulating the location related behavior of large grocery chains. Governments may use location regulation as a means to regulate competition.

Pricing regulation is hard to administer when stores sell a variety of products. The Australian government attempted to regulate prices of grocery chains by publishing the prices charged by each store for a basket of goods; this proved to be too costly and the website was shut down [13]. This may indicate that location regulation may be a more feasible way to ensure social welfare.

The question of price regulation is only binding under asymmetry, and hence prior to our analysis of asymmetric densities, price regulation could not be considered in any meaningful way ${ }^{3}$. There is of course more to the

\footnotetext{
${ }^{1}$ Taking as an example facility location (see [10] or [11]), firms have to tradeoff the transport costs of inputs and outputs in choosing both their location and production. Even in a non-strategic setting this problem is technically difficult and frequently requires solution by numerical operations research techniques.

${ }^{2}$ Total transport costs are the standard welfare measure, since under inelastic demand and identical production costs only transport costs impact on total surplus.

${ }^{3}$ We refer here to the regulation of a single market price, as opposed to price discrimination which, since [14], has been extensively considered in the literature.
} 
location of a firm than the stylized Hotelling model, but it is the workhorse of spatial competition and as we show the welfare implications are sensitive to the choice of consumer distribution.

\section{Social-0ptimum Location in the Quadratic Transport Cost Model}

We use a quadratic transport cost model as specified in [9], but generalize beyond uniform consumer density. There are two firms on the real line and each produces a homogeneous good at a constant marginal cost, henceforth normalized to zero. Firm $i$ is located at $x_{i}$, where $I=1,2$. Without loss of generality, the firm on the left will be denoted as firm 1 and the firm on the right will be firm 2, $x_{1} \leq x_{2}$. There is a continuum of consumers, whose mass is normalized to unity without loss of generality. The transport cost incurred by the consumer located at $x$ from consuming the good at $x_{i}$ is:

$$
t\left(x-x_{i}\right)^{2}
$$

where $t$ is the amount it costs to travel to purchase the good. For simplification, we let $t=1$.

The consumer density is $f(x), \geq$ for all $x$, and its cumulative distribution function is denoted by $F(x)$. We assume $f(x)$ is twice differentiable on its support $[a, b]$, where $a$ may be $-\infty$ and/or $b$ can be $\infty$.

The social-optimum problem is simply to minimize the aggregate transport costs, $T$, in serving consumers:

$$
T\left(x_{1}, x_{2}\right)=\int_{a}^{\mu}\left(x-x_{1}\right)^{2} f(x) \mathrm{d} x+\int_{\mu}^{b}\left(x-x_{2}\right)^{2} f(x) \mathrm{d} x,
$$

where $\mu$ is the boundary between the group of consumers who go to firm 1 and those who go to firm 2 . Since demand is inelastic and production costs are constant and identical across firms, the socially optimal value of $\mu$, denoted $\mu_{s}$, is

$$
\mu_{S}=\frac{1}{2}\left(x_{1}+x_{2}\right)
$$

since it is clearly optimal to serve each consumer from the closer firm.

Remark 1. As shown in [5], if $f(x)$ is logconcave then the first order conditions yields the unique socially optimal locations, i.e. the unique solution to the programme

$$
\min _{x_{1}, x_{2}} T\left(x_{1}, x_{2}\right) .
$$

\subsection{Logconcave Consumer Densities}

Given Remark 1, we naturally restrict attention in this paper to logconcave densities. Although logconcavity is sufficient for the existence of a unique pair of socially optimal location, additional conditions are required to guarantee existence of a unique competitive equilibrium, see [5]. Table 1 contains the list of distributions proven in [1] to satisfy the conditions for the existence of unique location-price equilibria.

\subsection{Social-0ptimum Locations}

A generic set of implicit conditions for the social-optimum locations, in terms of integral equations, is given in [5]. The pair of social-optimum locations (which minimizes $\left.T\left(x_{1}, x_{2}\right)\right)$ are the $\left(x_{1}^{S}, x_{2}^{S}\right)$ that solve:

$$
x_{1}^{S}=\mu_{S}-\int_{a}^{\mu_{S}} \frac{F(x)}{F\left(\mu_{S}\right)} \mathrm{d} x
$$

and

$$
x_{2}^{S}=\mu_{S}+\int_{\mu_{S}}^{b} \frac{1-F(x)}{1-F\left(\mu_{S}\right)} \mathrm{d} x,
$$

where $\mu_{S}$ solves

$$
\int_{a}^{\mu_{S}} \frac{F(x)}{F\left(\mu_{S}\right)} \mathrm{d} x=\int_{\mu_{S}}^{b} \frac{1-F(x)}{1-F\left(\mu_{S}\right)} \mathrm{d} x .
$$


Table 1. Standardized forms of the consumer densities considered.

\begin{tabular}{cccc}
\hline Density function & Support & Density function $f(x)$ & Cumulative distribution function $F(x)$ \\
\hline Uniform & {$[0,1]$} & 1 & $x$ \\
Normal & $(-\infty, \infty)$ & $\frac{1}{\sqrt{2 \pi}} \mathrm{e}^{-x^{2} / 2}$ & $\frac{1}{2}\left(\operatorname{erf}\left(\frac{1}{2} \sqrt{2} x\right)+1\right)$ \\
Logistic & $(-\infty, \infty)$ & $\frac{\mathrm{e}^{-x}}{\left(1+\mathrm{e}^{-x}\right)^{2}}$ & $\frac{1}{\left(1+\mathrm{e}^{-x}\right)^{2}}$ \\
Laplace & $(-\infty, \infty)$ & $\frac{1}{2} \mathrm{e}^{-|x|}$ & $\frac{1}{2}\left(1+\operatorname{sgn}(x)\left(1-\mathrm{e}^{-|x|}\right)\right)$ \\
Power $(c \geq 1)$ & {$[0,1]$} & $c x^{c-1}$ & $x^{c}$ \\
Weibull & {$[0, \infty)$} & $c x^{c-1} \mathrm{e}^{-x^{c}}$ & $1-\mathrm{e}^{-x^{c}}$ \\
Rayleigh & {$[0, \infty)$} & $2 x \mathrm{e}^{-x^{2}}$ & $1-\mathrm{e}^{-x^{2}}$ \\
\hline
\end{tabular}

Building on these results, we provide an explicit characterization of the socially optimal locations for symmetric distributions.

Proposition 1. For any symmetric, log-concave distribution with a mean/median of $M$ and mean-deviation $E(|X-M|)$, the social-optimum duopoly locations are one mean-deviation away from the median:

$$
x_{1}^{s}=M-E(|X-M|) \text { and } x_{2}^{s}=M+E(|X-M|) .
$$

Proof. Let $f(x)$ be a symmetric, log-concave probability density function with supports $(-b, b)$ and median $M=0$. [5] have shown that the socially optimal locations for duopoly firms are symmetric around the median for distribution $f(x)$. The distance between either firms and the median is

$$
\int_{M}^{b} \frac{1-F(x)}{1-F(M)} \mathrm{d} x
$$

To prove the proposition, we need to show that this distance equals one mean deviation or

$$
E(|X-M|)=\int_{M}^{b} \frac{1-F(x)}{1-F(M)} \mathrm{d} x .
$$

Since $X$ is symmetrically distributed, on the left-hand-side,

$$
E(|X-M|)=E(|X|)=\int_{-b}^{b}|x| f(x) \mathrm{d} x=2 \int_{0}^{b} x f(x) \mathrm{d} x .
$$

Integrating by parts gives

$$
2 \int_{0}^{b} x f(x) \mathrm{d} x=\left.2 x F(x)\right|_{0} ^{b}-2 \int_{0}^{b} F(x) \mathrm{d} x=2 b-2 \int_{0}^{b} F(x) \mathrm{d} x
$$

and on the right-hand-side,

$$
\int_{M}^{b} \frac{1-F(x)}{1-F(M)} \mathrm{d} x=2 \int_{0}^{b}[1-F(x)] \mathrm{d} x=2 \int_{0}^{b} \mathrm{~d} x-2 \int_{0}^{b} F(x) \mathrm{d} x=2 b-2 \int_{0}^{b} F(x) \mathrm{d} x,
$$

which establishes the result for $M=0$. Extension to $M \neq 0$ is trivial.

For log-concave densities, symmetry of the density is sufficient to make the socially optimal locations of the two firms symmetric about the median/mean of the consumer density. One might expect that as consumer locations become more dispersed, the locations of the two firms should also become further apart. We show in Proposition 3 on spread that this loose intuition is precisely true to the degree that dispersion of consumer preferences is measured by the mean deviation. With a formula for the socially optimal locations in hand, it is now 
possible to analyze the excess differentiation of the competitive locations compared to the socially optimal locations. Using results from Proposition 1, we solve the system of equations ${ }^{4}$ for each of the density functions which satisfy [5]'s three conditions (i.e. the distributions listed in Table 1) - the results are given in Proposition 2.

Proposition 2. The socially optimal locations and transport costs for five of the logconcave distributions given in Table 2, where $x_{i}^{S}$ 's are the social-optimum location for firm $i$, and $T^{S}$ 's are the social-optimum transportation costs.

Proof. See online working paper at: http://dx.doi.org/10.2139/ssrn.1615884.

The results for the Uniform case are already well known; the results for the other four distributions are new and allow for an analysis of spatial competition in the Hotelling framework which is no longer tied to the uniform distribution. The Rayleigh distribution is asymmetric and has socially optimal locations which are not symmetric about either its mean $(\approx 0.886)$ or its median $(\approx 0.833)$. Thus the symmetry result for the first four distributions in Proposition 1 does not hold in general for asymmetric densities. In the Rayleigh case the left firm is located in the dense left part of the distribution and the right firm is located well out into the long right tail which goes off to infinity. The locations are sufficiently to the right to place the boundary $\mu_{S}$ to the right of the median and the mean.

Table 2 shows that the optimal differentiation between the two firms varies considerably with the shape of the consumer density function and therefore the choice of density utilized in analyzing the Hotelling model could have a significant impact on the results. We benchmark the impact of the distribution shape below in two ways. First, in Section 3, we compare the socially optimal locations with the competitive equilibrium locations and find how savings on transportation costs varies across distributions. Second, in Section 4, we go beyond the standardized functional forms above to compare the social optimum results for different distributions as the dispersion of the distributions increases.

The "standardized" versions of the Power and Weibull distributions depend on the parameter $c$, and as a result the socially optimal locations also depend on $c$. The more involved results for these two families are presented in the next section.

\subsection{Special Cases: Asymmetric Distribution}

Next, we look at the social optimum locations of two asymmetric families of distributions, specifically, the Weibull and the Power distributions.

\subsubsection{The Weibull Distribution}

The Weibull distribution has no explicit solution for $\mu_{S}$. In this section, we look at the solution to the Weibull distribution in detail. $\mu_{S}$ for the Weibull distribution solves the following equation:

$$
\begin{aligned}
& \int_{0}^{\mu_{S}} \frac{F(x)}{F\left(\mu_{S}\right)} \mathrm{d} x=\int_{\mu_{S}}^{\infty} \frac{1-F(x)}{1-F\left(\mu_{S}\right)} \mathrm{d} x \\
& \Rightarrow \frac{1}{\mathrm{e}^{-\mu_{S}^{c}}-1} \int_{0}^{\mu_{S}}\left(\mathrm{e}^{-x^{c}}-1\right) \mathrm{d} x=\mathrm{e}^{\mu_{S}^{c}} \int_{\mu_{S}}^{\infty} \mathrm{e}^{-x^{c}} \mathrm{~d} x
\end{aligned}
$$

Equation (Weibull) involves the integral of $\mathrm{e}^{-x^{c}}$ of which the closed form cannot be determined for $c>2$. The Exponential and the Rayleigh distributions are the Weibull with $c=1$ and $c=1$ respectively. For the exponential distribution, we find that $x_{1}^{S}=0.5936, x_{2}^{S}=2.5936$ and $\mu_{S}=1.5936$. However, as proven in [1], the Exponential distribution does not satisfy the conditions for the existence of unique location-price equilibria. Thus, for the class of Weibull distributions, only the Rayleigh distribution will be discussed in the subsequent sections. For the Rayleigh distribution, we find that $\mu_{S}=0.97213$, and the social-optimum locations are $x_{1}^{S}=0.58634, x_{2}^{S}=1.3579$.

\subsubsection{The Power Distribution}

Similarly, there is no explicit solution for the Power distribution either. Here, we find $\mu_{S}$ that solves the integral equation for the Power distribution:

\footnotetext{
${ }^{4}$ Equations (5), (6) and (7).
} 
Table 2. Social-optimum locations for density functions which satisfy the conditions in [5].

\begin{tabular}{ccccc}
\hline Density Function & $\mu_{S}$ & $x_{1}^{s}$ & $x_{2}^{s}$ & $T^{s}$ \\
\hline Uniform & 0.5 & 0.25 & 0.75 & 0.02083 \\
Normal & 0 & -0.7979 & 0.7979 & 0.3634 \\
Logistic & 0 & -1.3863 & 1.3863 & 1.3863 \\
Laplace & 0 & -1 & 1.358 & 1 \\
Rayleigh & 0.9721 & 0.5863 & & 0.07315 \\
\hline
\end{tabular}

$$
\begin{aligned}
& \int_{0}^{\mu_{S}} \frac{F(x)}{F\left(\mu_{S}\right)} \mathrm{d} x=\int_{\mu_{S}}^{\infty} \frac{1-F(x)}{1-F\left(\mu_{S}\right)} \mathrm{d} x \Rightarrow \frac{1}{\mu_{S}^{c}} \int_{0}^{\mu_{S}} x^{c} \mathrm{~d} x=\frac{1}{\mu_{S}^{c}-1} \int_{\mu_{S}}^{\infty}\left(x^{c}-1\right) \mathrm{d} x \\
& \Rightarrow \mu_{S}=\frac{c+1}{1-\mu_{S}^{c}}\left(1-\mu_{S}\right)+\frac{\mu_{S}^{c+1}-1}{1-\mu_{S}^{c}}
\end{aligned}
$$

The above can only be solved explicitly for fixed levels of $c$. Hence the value of $\mu_{S}$ depends on $c$. To examine the relationship between $\mu_{S}$ and $c$, the following total differential equation is obtained.

$$
\mathrm{d} \mu_{\mathrm{S}}=-\mu_{\mathrm{S}}\left(\mu_{\mathrm{S}}-1\right) \frac{c \mu_{\mathrm{S}}^{c} \ln \mu_{\mathrm{S}}-\mu_{\mathrm{S}}^{c}+1}{2 \mu_{\mathrm{S}}-c^{2} \mu_{S}^{c}+c \mu_{S}+2 \mu_{S}^{2 c+1}-4 \mu_{S}^{c+1}+c^{2} \mu_{S}^{c+1}-c \mu_{S}^{c+1}} \mathrm{~d} c
$$

The first part on the right-hand-side, $-\mu_{S}\left(\mu_{S}-1\right)$, is positive. To determine the sign of the second part, we check the first order conditions and concavity of the numerator and denominator. The result shows that both functions are positive given the constraints $c \geq 1, \mu_{S} \in[0.5,1]$ and Equation (15) holds. Therefore, the value of $\mu_{S}$ increases with $c$, where $c$ determines the curvature of Power distribution's density function.

Knowing the direction of change of $\mu_{S}$ resulted from a change in $c$, we can now derive the change in $x_{1}^{S}$ and $x_{2}^{S}$ from Equations (5) and (6). The deviation of firms' location from $\mu_{S}$ is

$$
\int_{a}^{\mu_{S}} \frac{F(x)}{F\left(\mu_{S}\right)} \mathrm{d} x=\frac{\mu_{S}}{c+1}
$$

A check on the second order conditions for $\mathrm{d} \mu_{S} / \mathrm{d} c$ shows that it is a concave function with maximum value at $\mu_{S}=0.5$ and $c=1$, i.e. $\max _{\left(\mu_{S}, c\right)} \frac{\mathrm{d} \mu_{S}}{\mathrm{~d} c}=0.153$. We then have

$$
\frac{\mu_{S}+\Delta \mu_{S}}{c+\Delta c+1}<\frac{\mu_{S}}{c+1}
$$

since the denominator increases at a faster rate than the numerator. From here we know that the deviation of firms' locations from $\mu_{S}$ decreases as $\mu_{S}$ approaches 1 . As such, product differentiation decreases as the curvature of the density function increases.

To illustrate, we plot the graph of $\mu_{S}$ against $c$ and solve numerically for Power distributions with integer values $2 \leq c \leq 10$. The graph and values are in Figure 1 and Table 3 respectively.

It can be observed that $\mu_{S}$ is increasing in $c$ and the two socially optimum locations get closer as $c$ increases. However the increase of the market boundary for firm 1 does not translate in to an increase in market share for firm 1 because the density is also changing shape in $c$. For example, at $c=2$, firm 1 serves $38.2 \%$ of the market while at $c=10$ firm 1's market share falls to $24.5 \%$. The amount of differentiation will be discussed in detail in the next section.

\section{Equilibrium and Social-0ptimum Differentiation}

[1] derive the bounds for the ratio between social-optimum and equilibrium differentiation for symmetric distributions. Specifically they find 
Table 3. The social-optimal locations under the Power distribution.

\begin{tabular}{cccc}
\hline$c$ & $\mu_{S}$ & $x_{1}^{s}$ & $x_{2}^{s}$ \\
\hline 2 & 0.6180 & 0.4120 & 0.8240 \\
3 & 0.6914 & 0.5186 & 0.8643 \\
4 & 0.7413 & 0.5930 & 0.8895 \\
5 & 0.7773 & 0.6478 & 0.9069 \\
6 & 0.8046 & 0.6896 & 0.9195 \\
7 & 0.8259 & 0.7226 & 0.9291 \\
8 & 0.8430 & 0.7493 & 0.9367 \\
9 & 0.8571 & 0.7714 & 0.9428 \\
\hline
\end{tabular}

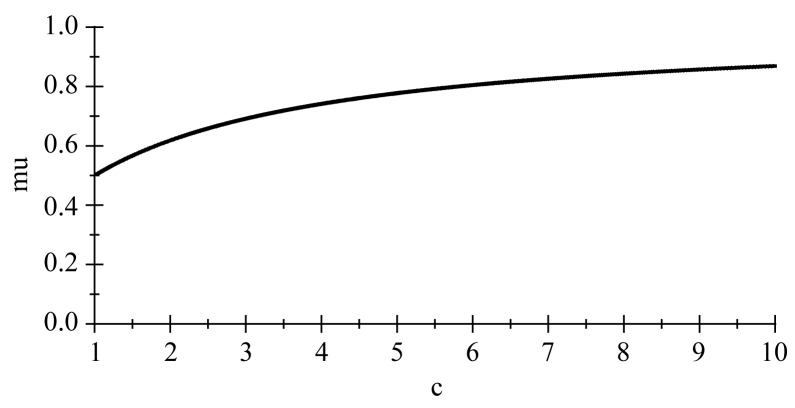

Figure 1. Graph of $\mu_{S}$ against $c$.

$$
\frac{1}{3} \leq R \leq \frac{2}{3}
$$

where

$$
R=\frac{x_{2}^{S}-x_{1}^{S}}{x_{2}^{*}-x_{1}^{*}}=\frac{\Delta x^{S}}{\Delta x^{*}} .
$$

$R$ is inversely proportional to the amount of excess differentiation. These bounds show there is always substantial excess differentiation in the location-price game [5], also show that increasing the dispersion of consumers (in the form below) increases the equilibrium differentiation ${ }^{5}$. In contrast, we now show that the excess differentiation ratio is invariant to this kind of increase in the dispersion of consumers.

Proposition 3. If consumers are distributed according to a continuous, log-concave, symmetric density $f$ on $[M-b, M+b]$ which satisfies the technical restrictions of $[5]^{6}$ then

$$
R(f) \equiv \frac{\Delta x^{s}}{\Delta x^{*}}=\frac{4}{3} E(|X-M|) f(M)
$$

and the differentiation ratio, $R\left(f_{\sigma}\right)$, is invariant to the mean preserving spreads of the form $f(x-M)=\frac{1}{\sigma} f\left(\frac{x-M}{\sigma}\right)$, with $\sigma>0$, i.e. $\frac{\mathrm{d} R\left(f_{\sigma}\right)}{\mathrm{d} \sigma}=0$.

Proof. From [5], the conditions on $f$ give $\Delta x^{*}=\frac{3}{2 f(M)}$. The expression for $R$ then follows from direct

${ }^{5}$ Subsequently, [1] compared the Uniform, Normal and Logistic for the sensitivity of location to changes in variance, as they are most commonly used to describe populations.

${ }^{6}$ These technical restrictions are important because symmetric densities do not necessarily have symmetric location equilibria, see [6]. 
substitution of the previous proposition:

$$
\frac{\Delta x^{s}}{\Delta x^{*}}=\frac{2 E(|X-M|)}{\frac{3}{2 f(M)}}=\frac{4}{3} E(|X-M|) f(M) .
$$

Using this explicit expression we now derive the invariance result, without loss of generality assume $M=0$. Let $E_{\sigma}$ denote the expectation with regard to the distribution $f_{\sigma}$, then

$$
E_{\sigma}(|X|)=2 \int_{0}^{\sigma b} y f_{\sigma}(y) \mathrm{d} y=2 \int_{0}^{\sigma b} y f\left(\frac{y}{\sigma}\right) \frac{1}{\sigma} \mathrm{d} y=2 \int_{0}^{b} z \sigma f(z) \frac{1}{\sigma} \sigma \mathrm{d} z=\sigma E(|X|) .
$$

where $z=y / \sigma$. Simple substitution gives the result with $M \neq 0$. Thus for the mean preserving spread given by $f_{\sigma}$ we have

$$
R\left(f_{\sigma}\right)=\frac{4}{3} E_{\sigma}(|X-M|) f_{\sigma}(M)=\frac{4}{3} \sigma E(|X|) \frac{1}{\sigma} f\left(\frac{0}{\sigma}\right)=R(f) .
$$

For this type of mean preserving spread, the increase in the absolute deviation from the median/mean is linear in $\sigma$ while the decrease in the density of the mean is at the rate $1 / \sigma$, so the two effects exactly balance. That is, in this case the tendency for socially optimal locations to increase because of greater dispersion is proportionally the same as the tendency for competitive locations to move apart due to decreased competition for the marginal consumer at $M$.

The exact amount of differentiation that each distribution results in is shown in Table 4 and Table 5. As [5] observe that amongst the symmetric distributions the Uniform and Laplace distributions exhibit the greatest and least divergence respectively. However, in extending the analysis to asymmetric distributions, we see that asymmetric distributions have greater divergence than any of the symmetric distributions in the group.

For the Power distribution, under a duopoly location-then-price game, the product differentiation ${ }^{7}$ is

$$
\Delta x^{*}=\left(\frac{1}{c+1}\right)^{\frac{1}{c}}\left(\frac{2 c+c^{2}+2}{c(c+1)}+\frac{c}{c+1}\right)=\frac{2}{c} \frac{\left(\frac{1}{c+1}\right)^{\frac{1}{c}}}{c+1}\left(c^{2}+c+1\right) .
$$

The first derivative is

$$
\frac{\mathrm{d} \Delta x^{*}}{\mathrm{~d} c}=\frac{1}{2} \frac{c}{\left(\frac{1}{c+1}\right)^{\frac{1}{c}}} \frac{c+1}{c^{2}+c+1}>0,
$$

\begin{tabular}{|c|c|c|c|}
\hline Density Function & $x_{2}^{S}-x_{1}^{S}=\Delta x^{S}$ & $x_{2}^{*}-x_{1}^{*}=\Delta x^{*}$ & $R=\Delta x^{\mathrm{s}} / \Delta x^{*}$ \\
\hline Uniform & 0.5 & 1.5 & 0.333 \\
\hline Normal & $\frac{2 \sqrt{2}}{\sqrt{\pi}}=1.596$ & $\frac{3}{2} \sqrt{2} \sqrt{\pi}=3.760$ & $\frac{4}{3 \pi}=0.424$ \\
\hline Logistic & $4 \ln 2=2.773$ & 6 & $2 / 3 \ln 2=0.462$ \\
\hline Laplace & 2 & 3 & $2 / 3=0.667$ \\
\hline Power $(c \geq 1)$ & & Depend on $c$ (see Table 5) & \\
\hline Rayleigh & 0.772 & 2.496 & 0.309 \\
\hline
\end{tabular}

so the amount of product differentiation increases as $c$ increases. Examples are given in Table 5. Since the product differentiation increases under equilibrium and decreases under social-optimum conditions, the amount

Table 4. Amount of differentiation $\left(x_{2}-x_{1}\right)$ for equilibrium and social-optimum locations and their ratios.

\footnotetext{
${ }^{7}$ See online working paper at: http://dx.doi.org/10.2139/ssrn.1615884.
} 
of divergence, $R$, increases with $c$.

As $c$ increases for the Power distribution, it can be observed that the optimum amount of product differentiation decreases while the equilibrium amount of differentiation increases. The divergence between the two gets larger as $c$ increases. The need for government intervention may increase as the distribution gets more skewed (Figure 2).

\section{Savings from Optimum Allocation}

The social-optimum locations are transport cost minimizing; there will be a saving on transport cost if the socialoptimum locations are used instead of the equilibrium. The differences in transportation costs between equilibrium and social-optimum outcomes show the savings in transport cost and are detailed in Table 6 and Table 7. We introduce a saving ratio $(S)$ which is calculated as savings over transport costs (incurred with the equilibrium locations)

$$
S=\left|\frac{T^{*}-T^{S}}{T^{*}}\right| .
$$

The Power distribution, having the greatest divergence of equilibrium from optimum, has the greatest saving ratio. Whereas, the Laplace distribution, having the least divergence, exhibits the lowest saving ratio. The saving ratios imply that in a two stage location-price game, the Laplace distribution results in a cost closest to the social-optimum. On the other hand, when moving from equilibrium to social-optimum locations, it is most efficient in cost-reduction under the Power distribution. We can infer a negative relationship between $R$ and the

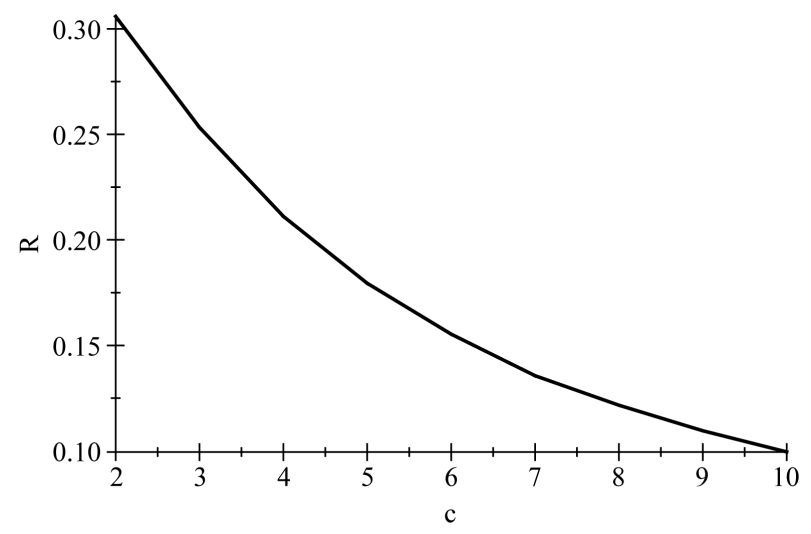

Figure 2. Plot of $R$ against $c$ for power distribution.

Table 5. Amount of differentiation $\left(x_{2}-x_{1}\right)$ and their ratios for the Power distribution.

\begin{tabular}{cccc}
\hline$c$ & $x_{2}^{s}-x_{1}^{s}=\Delta x^{s}$ & $x_{2}^{*}-x_{1}^{*}=\Delta x^{*}$ & $R=\Delta x^{s} / \Delta x^{*}$ \\
\hline 2 & 0.4120 & 1.3472 & 0.3059 \\
3 & 0.3457 & 1.3649 & 0.2533 \\
4 & 0.2965 & 1.4044 & 0.2111 \\
5 & 0.2591 & 1.4442 & 0.1794 \\
6 & 0.2299 & 1.4805 & 0.1553 \\
7 & 0.2065 & 1.5125 & 0.1365 \\
8 & 0.1973 & 1.5408 & 0.1216 \\
9 & 0.1714 & 1.5657 & 0.1095 \\
10 & 0.1580 & 1.5879 & 0.0995 \\
\hline
\end{tabular}


Table 6. Savings and saving ratios for the set of density functions.

\begin{tabular}{ccccc}
\hline Density Function & $T^{\varsigma}$ & $T^{*}$ & $T^{*}-T^{\varsigma}$ & Savings Ratio $(S)$ \\
\hline Uniform & 0.021 & 0.271 & 0.250 & 0.923 \\
Normal & 0.363 & 1.534 & 1.171 & 0.763 \\
Logistic & 1.368 & 3.972 & 2.604 & 0.656 \\
Laplace & 1 & 1.25 & 0.25 & 0.200 \\
Power (c $\geq 1)$ & & Depend on $c$ (see Table 7) & 0.907 \\
Rayleigh & 0.07315 & 0.7885 & 0.7154 & \\
\hline
\end{tabular}

Table 7. Savings and saving ratios for Power distribution with integers $c$ such that $2 \leq c \leq 10$.

\begin{tabular}{ccccc}
\hline$c$ & $T^{\varsigma}$ & $T^{*}$ & $T^{*}-T^{\varsigma}$ & Savings Ratio $(S)$ \\
\hline 2 & 0.0155 & 0.2293 & 0.2139 & 0.9325 \\
3 & 0.0111 & 0.2369 & 0.2259 & 0.9533 \\
4 & 0.0081 & 0.2381 & 0.2300 & 0.9658 \\
5 & 0.0062 & 0.2337 & 0.2275 & 0.9735 \\
6 & 0.0049 & 0.2265 & 0.2216 & 0.9785 \\
7 & 0.0039 & 0.2180 & 0.2141 & 0.9821 \\
8 & 0.0032 & 0.2092 & 0.2060 & 0.9847 \\
9 & 0.0027 & 0.2004 & 0.1977 & 0.9866 \\
\hline
\end{tabular}

saving ratio.

The savings ratio does not reflect the effect on producers as equilibrium prices are different for both sets of locations. Take for example the uniform distribution, the equilibrium locations are $(-1 / 4,5 / 4)$ and price is $3 / 2$ (thus total profits are $3 / 2$ ), social optimum locations are $(1 / 4,3 / 4$ ) and price is $1 / 2$ (total profits are $1 / 2$ ). Although overall welfare is improved (net welfare improvement is $1 / 4$, see Table 6 ), the loss in total profits from changing to the social-optimum location is 1 . This fall in profits is due to the decrease in product differentiation. A larger $R$ would mean less excessive differentiation and thus a smaller drop in profits if firms are made to locate at the social optimal locations.

\section{The Impact of Consumer Dispersion}

In [5], it was determined that variance affects the equilibrium outcome of the location game. Subsequently, [1] compared the Uniform, Normal and Logistic for the sensitivity of location to changes in variance, as they are most commonly used to describe populations. Figure 3 shows the effect of variance on the amount of differentiation in the social optimum outcomes.

We find that the socially optimum amount of differentiation is the most sensitive to variance under the Uniform distribution; distance between firms increase at a faster rate compared to the Logistic and the Normal distributions. The Logistic is the least sensitive to changes in variance. This result is consistent with the equilibrium analysis in [1].

We calculate $R$ to test the effects of changes in variance on the amount of divergence of equilibrium from optimum, Table 8 contains these values. As derived in Proposition 3, variance does not have any effect on the amount of divergence of the equilibrium from the optimum $(R)$. The amount of divergence from the equilibrium is largest for the logistic distribution and smallest for the uniform distribution. 


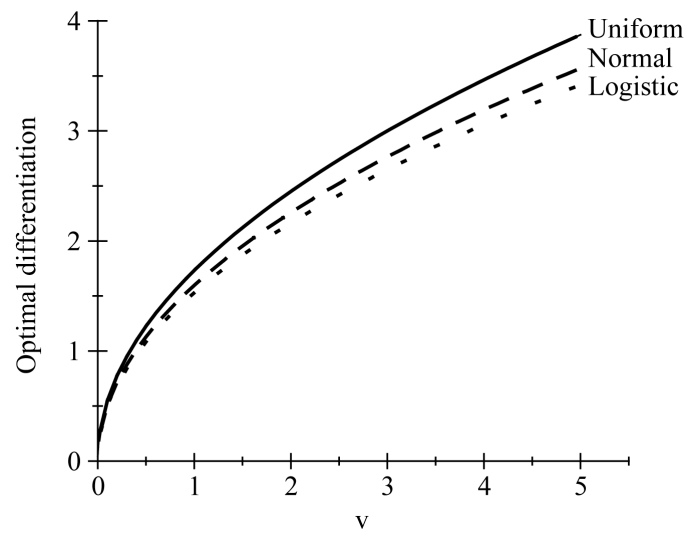

Figure 3. Distance between firms and variance $(v)$.

Table 8. General form: social-optimum locations.

\begin{tabular}{cccc}
\hline Distribution Variables & Uniform & Normal & Logistic \\
\hline$v$ & $b^{2} / 12$ & $\sigma^{2}$ & $\pi^{2} b^{2} / 3$ \\
$\mu_{S}$ & $1 / 2 b$ & 0 & 0 \\
$x_{1}^{S}$ & $1 / 4 b$ & $-\frac{\sqrt{2}}{\sqrt{\pi}} \sigma$ & $-2 b \ln 2$ \\
$x_{2}^{S}$ & $3 / 4 b$ & $\frac{\sqrt{2}}{\sqrt{\pi}} \sigma$ & $2 b \ln 2$ \\
$D^{S}$ & $\sqrt{3} \sqrt{v}$ & $\frac{2 \sqrt{2}}{\sqrt{\pi}} \sqrt{v}$ & $4 \ln 2 \sqrt{\frac{3 v}{\pi^{2}}}$ \\
$D^{*}$ & 18 & $\frac{3}{2} \sqrt{2} \sqrt{\pi} \sqrt{v}$ & $6 \sqrt{\frac{3 v}{\pi^{2}}}$ \\
\hline
\end{tabular}

Note: the values of $D^{*}$ are found in [1].

\section{The Duopoly Price Game at Social-Optimum Locations}

\subsection{Equilibrium of the Price Game}

The social-optimum conditions require the cost of transportation to be minimal, and thus consumers should only buy goods from whichever firm is closer to them. However, consumers' decisions do not depend only on the cost of transportation, but also on the prices charged by the firms. A consumer located at $x$ will buy one unit of the good from the firm, where the following mill price,

$$
p_{i}+\left(x-x_{i}^{S}\right)^{2}
$$

is lower. $p_{i}$ is the price charged by firm $i$. From [5], given the location combination $\left(x_{1}^{S}, x_{2}^{S}\right)$, firms maximize profits by setting prices at:

$$
p_{1}^{*}=2\left(x_{2}^{S}-x_{1}^{S}\right) \frac{F\left(\xi^{S}\right)}{f\left(\xi^{S}\right)},
$$

and

$$
p_{2}^{*}=2\left(x_{2}^{S}-x_{1}^{S}\right) \frac{1-F\left(\xi^{S}\right)}{f\left(\xi^{S}\right)},
$$


where $\xi$ is the indifferent consumer uniquely determined by the equality of full prices, $p_{1}+\left(x^{S}-x_{1}^{S}\right)^{2}=p_{2}+\left(x^{S}-x_{2}^{S}\right)^{2}$. Hence,

$$
\xi^{S}=\frac{1}{2}\left(x_{1}^{S}+x_{2}^{S}+\frac{p_{2}-p_{1}}{\left(x_{2}^{S}-x_{1}^{S}\right)}\right) .
$$

To minimize the transportation cost, the indifferent consumer must be at the mid-point between $x_{1}$ and $x_{2}$, i.e. $x^{S}=\mu_{S}=1 / 2\left(x_{1}^{S}+x_{2}^{S}\right)$. This requires both firms to charge equal price, i.e. $p_{1}=p_{2}$. We check if the location combination $\left(x_{1}^{S}, x_{2}^{S}\right)$ support equal price for each of the distributions mentioned previously. For any given symmetric density function, the prices of the two firms will be equal ${ }^{8}$. This is not true for asymmetric distributions, such as the Power and Rayleigh distributions.

\subsection{Social-Optimum Locations of Asymmetric Distributions}

In this section, we pay particular attention to the asymmetric distributions. Due to the asymmetric nature of the consumers' distribution, firms do not set equal prices when they are at the social-optimum locations, thus causing deviation from cost minimization. The following sections explain this in detail.

\subsubsection{Social-Optimum Outcome for Asymmetric Distributions}

Lemma 1. For any single-mode asymmetric distribution with median $M, M \neq \mu_{S}$, where $\mu_{S}$ is the indifferent consumer location under social optimality (from Equation (7)). The indifferent consumer is not located at the median.

Proof. We prove this lemma by contradiction. Assuming that $M$ is a solution to Equation (7), we have the following:

$$
\int_{a}^{M} \frac{F(x)}{F(M)} \mathrm{d} x=\int_{M}^{b} \frac{1-F(x)}{1-F(M)} \mathrm{d} x \Rightarrow 2 \int_{a}^{M} F(x) \mathrm{d} x=2 \int_{M}^{b} 1-F(x) \mathrm{d} x .
$$

Rearranging terms, we have:

$$
\int_{a}^{M} F(x) \mathrm{d} x+\int_{M}^{b} F(x) \mathrm{d} x=\int_{M}^{b} \mathrm{~d} x \Rightarrow \int_{a}^{b} F(x) \mathrm{d} x=b-M .
$$

Again, using integration by parts on the left hand side:

$$
\left.F(x) x\right|_{a} ^{b}-\int_{a}^{b} x f(x) \mathrm{d} x=b-M \Rightarrow b-E[X]=b-M .
$$

However, for any asymmetric distribution with single mode, $E(X) \neq M$. Equation (35) contradicts our assumption that $X$ has an asymmetric density function. As such, $M$ is not a solution to Equation (7).

Lemma 2. If prices are equal in the location-then-price duopoly game, the indifferent consumer is located at the median and the locations of the firms are symmetric about the median.

Proof. Suppose the consumers are distributed with median $M$. According to [5], in a duopoly price game, the equilibrium prices, $p_{i}(i=1,2)$, and the indifferent consumer, $\xi$, are determined by

$$
\begin{gathered}
p_{1}=2\left(x_{2}-x_{1}\right) \frac{F(\xi)}{f(\xi)}, \\
p_{2}=2\left(x_{2}-x_{1}\right) \frac{1-F(\xi)}{f(\xi)}, \\
\xi=\frac{1}{2}\left(x_{1}+x_{2}\right)+\frac{1-2 F(\xi)}{f(\xi)} .
\end{gathered}
$$

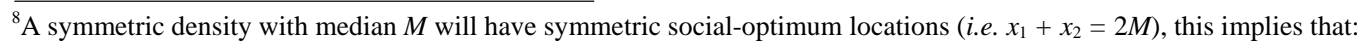

$$
p_{1}=p_{2}=\frac{x_{2}-x_{1}}{f(M)} \text {. }
$$

Therefore, if firms were given the freedom to set prices, they will set equal prices which can be calculated by using (equal price). 
From Equations (36) and (37), equating $p_{1}$ and $p_{2}$, we have $F(\xi)=0.5$, and hence $\xi=M$. In addition, substituting $\xi=M$ into Equation (38), we have $\xi=1 / 2\left(x_{1}+x_{2}\right)$. Therefore, the locations of the two firms are symmetric about the indifferent consumer when the prices are equal.

Having proven the above two lemmas, we are now ready to prove the following proposition.

Proposition 4. The social optimum cannot be achieved by sole regulation of locations if consumer distributions are asymmetric. Prices regulations are also required.

Proof. Consider first the case of socially optimal locations $\left(x_{1}^{S}, x_{2}^{S}\right)$ and competitive prices. By Lemma 1 , the median $M$ is not a solution (for asymmetric distributions) to Equation (7), i.e. $M \neq \mu_{\text {s. }}$. The social-optimum locations are symmetric about $\mu_{S}$ as implied by Equations (5), (6) and (7). Hence these social-optimum locations are not symmetric about the median $M$. Taking the contrapositive of Lemma 2 implies that firms' competitive prices are not identical at the socially optimal locations. Next, we show that this set of locations with non-equal prices are not welfare maximizing.

The indifferent consumer, $\xi$, is not at $1 / 2\left(x_{1}+x_{2}\right)$. Suppose $\xi^{\xi}>\mu_{S}$, consumers located at the interval $\left(\mu_{S}, \xi\right)$ would pay a higher transportation cost to buy goods from firm 1 while it incurs less cost to buy the same goods from firm 2. On the other hand, if $\xi^{S}<\mu_{S}$, buying goods from firm 2 would incur higher transportation cost for consumers in the interval $\left(\xi, \mu_{S}\right)$. The transportation costs spent by consumers in the interval $\left(\min \left(\xi \delta, \mu_{S}\right)\right.$, $\left.\max \left(\xi^{S}, \mu_{S}\right)\right)$ are not minimized while that spent by all the other consumers are. We then arrive at the conclusion that the society's transportation costs are higher when $\xi^{S}$ is different from $\mu_{\mathrm{s}}$. Thus, we have shown that the sole regulation of locations do not eventually result in transportation cost minimization. What happens if prices are regulated too?

Suppose the firms are required to set equal price, $p_{1}^{S}=p_{2}^{S}$. Equations (36) and (37) can be disregarded as firms do not have the freedom to set the prices. According to Equation (31), the indifferent consumer, $\xi$, is now located at $1 / 2\left(x_{1}+x_{2}\right)$, which is exactly the location of $\mu_{s}$. All consumers would then buy goods from whichever firm that is closer to them. The society's total transportation cost is minimized as each single consumer's transportation cost is minimized. That completes the proof of the proposition.

In the following sections, we discuss two examples of asymmetric distributions which appear in the previous parts of the paper (these are distributions where unique pure strategy equilibrium exists). They are the Power and the Rayleigh distributions.

\subsubsection{The Power Distribution}

We examine a subset of this distribution, where $2 \leq c \leq 10$. Equations (29), (30) and (31) are applied to solve $\xi$, $p_{1}^{S}$ and $p_{2}^{S}$. The results are listed in Table 9 .

Comparing Table 3 and Table 9, one finds that if firms at the social-optimum locations were given the freedom to set their own prices, the indifferent consumer, $\xi^{S}$, deviates from $\mu_{s}$. Comparing Table 7 with Table 9, one finds that savings ratio has fallen for all cases, recall that $S=\left|\frac{T^{*}-T^{S}}{T^{*}}\right|$, this denotes the savings in transport costs as a result of switching from the equilibrium locations to the social-optimal. In Table 7 firms are assumed to set equal prices, in Table 9 firms are allowed to set their own price; as a consequence, there is less savings in transport costs. For integer values of $c \in[2,10], \xi^{S}>\mu_{S}$. This means consumers located at the interval $\left(\mu_{S}, \xi^{s}\right)$ would buy goods from firm 1 while it incurs less transportation cost to buy from firm 2 . Therefore, the society's costs are not minimized in this case.

\subsubsection{The Rayleigh Distribution}

Similarly for the Rayleigh distribution, we apply Equations (29), (30) and (31) to solve $\xi$, $p_{1}^{S}$ and $p_{2}^{S}$. The resulting indifferent consumer is at $x=0.8787$, which is on the left of $\mu_{S}$. Consumers located at $0.8787<x<$ 0.9721 would buy goods from firm 2 while it incurs less transportation cost to buy from firm 1 . The transportation costs for consumers in this interval are not minimized, and hence society's total transportation cost is not the minimum. The saving ratio $(S)$ for the Rayleigh distribution is 0.900 and is smaller than when the firms are forced to set equal prices where $S=0.907$.

\section{Conclusions}

In [5], an implicit system of equations was provided which we build upon to give an explicit closed form solu- 
Table 9. Social-optimal attributes for the Power distribution.

\begin{tabular}{ccccc}
\hline$c$ & $\xi$ & $p_{1}^{s}$ & $p_{2}^{s}$ & Savings Ratio $(S)$ \\
\hline 2 & 0.67784 & 0.27928 & 0.32856 & 0.92405 \\
3 & 0.76058 & 0.17529 & 0.22311 & 0.94195 \\
4 & 0.80898 & 0.11993 & 0.16008 & 0.95471 \\
5 & 0.84091 & 0.08715 & 0.12011 & 0.96335 \\
6 & 0.86361 & 0.06618 & 0.09333 & 0.96942 \\
7 & 0.88061 & 0.05195 & 0.07455 & 0.97387 \\
8 & 0.89382 & 0.04186 & 0.06090 & 0.97726 \\
9 & 0.90438 & 0.03445 & 0.05067 & 0.97990 \\
\hline
\end{tabular}

tion for the social-optimum locations for symmetric consumer densities-each firm should be located one meandeviation away from the median. With this result we are also able to provide an explicit formula for the ratio of excess differentiation $R$ (socially optimal differentiation relative to equilibrium differentiation). We show that the excess differentiation ratio is invariant to a multiplicative increase in the dispersion of consumers around their mean/median.

We derive the social-optimum locations and prices for densities with unique pure strategy equilibria in a twostage location-price game and examine the degree of excess product differentiation. The results show that firms under the Uniform and Rayleigh densities have the greatest excess differentiation at their equilibrium locations.

This paper also looked at the potential saving from the regulation of firm decisions via the calculation of the savings ratio $(S)$ in Section 3.1. We found that the Laplace has the lowest $S$ and thus give governments the least incentive to intervene. The Power, Uniform and Rayleigh distributions exhibit high savings ratios and would benefit the most from government intervention. Policy makers and urban planners should consider consumer distributions when deciding to regulate firm location decisions.

The implicit social-optimum locations provided by [5] apply to both symmetric and asymmetric densities as they are transport cost minimizing. But the solution does not take into account the redistributive effect of prices in the pricing stage. The social-optimal locations for symmetric densities are chosen such that firms will naturally choose equal prices at the pricing stage. As long as prices are equal, the indifferent consumer remains at the median and transportation costs are minimized. On the other hand, under asymmetric densities (such as the Rayleigh distribution) firms will not choose equal prices (at the pricing stage) due to the asymmetric nature of the distribution. When prices are unequal, more consumers go to the cheaper firm (than socially optimal); this will cause transportation costs to deviate from the minimum. Thus, the sole regulation of location will not work for asymmetric densities as firms will not choose symmetric prices. This problem can be tackled by price regulation or one can work out an alternative location solution which will minimize transport costs where firms can set their own prices. The latter is a question open for future research.

\section{References}

[1] Meagher, K.J., Teo, E.G.S. and Wang, W. (2008) A Duopoly Location Toolkit: Consumer Densities Which Yield Unique Spatial Duopoly Equilibria. The B.E. Journal of Theoretical Economics, 8, Article 14.

[2] Fujita, M. and Thisse, J.-F. (2002) Economics of Agglomeration: Cities, Industrial Location, and Regional Growth. Cambridge University Press, Cambridge. http://dx.doi.org/10.1017/CBO9780511805660

[3] Caplin, A. and Nalebuff, B. (1991) Aggregation and Imperfect Competition: On the Existence of Equilibrium. Econometrica, 59, 1-23. http://dx.doi.org/10.2307/2938238

[4] Dierker, E. (1991) Competition for Customers. In: Cornet, B., d’Aspremont, C. and Mas-Collel, A., Eds., Equilibrium Theory and Applications, Cambridge University Press, Cambridge, 383-402.

[5] Anderson, S.P., Goeree, J.K. and Ramer, R. (1997) Location, Location, Location. Journal of Economic Theory, 77, 102-127. http://dx.doi.org/10.1006/jeth.1997.2323 
[6] Tabuchi, K. and Thisse, J.-F. (1995) Asymmetric Equilibria in Spatial Competition. International Journal of Industrial Organization, 13, 213-227. http://dx.doi.org/10.1016/0167-7187(94)00449-C

[7] Scrimitore, M. (2003) Symmetric and Asymmetric Equilibria in a Spatial Duopoly. Dattiloscritto, Lecce.

[8] Meagher, K. and Zauner, K.G. (2011) Uncertain Spatial Demand and Price Flexibility: A State Space Approach to Duopoly. Economic Letters, 113, 26-28. http://dx.doi.org/10.1016/j.econlet.2011.06.003

[9] d’Aspremont, C., Gabszewicz, J.J. and Thisse, J.F. (1979) On Hotelling’s “Stability in Competition”. Econometrica, 47, 1145-1150. http://dx.doi.org/10.2307/1911955

[10] Hurter, A.P. and Martinich, J.S. (1989) Facility Location and the Theory of Production. Kluwer Academic Publishers, Boston. http://dx.doi.org/10.1007/978-94-009-2518-2

[11] Peeters, D. and Thisse, J.-F. (2000) The Production-Location Problem Revisited. Papers in Regional Science, 79, 221-231. http://dx.doi.org/10.1007/s101100050044

[12] Competition Commission, United Kingdom (2008) Groceries Market Investigation, Provisional Decision on Remedies: Background and Overall Assessment. Competition Commission, United Kingdom. http://www.competition-commission.org.uk/inquiries/ref2006/grocery/pdf/decision_remedies_background.pdf

[13] Senate Economics References Committee, Commonwealth of Australia (2009) GROCERY Choice Website, Senate Printing Unit, Parliament House, Canberra. http://www.aph.gov.au/senate/committee/economics ctte/grocery choice 09/report/

[14] Lederer, P.J. and Hurter, A.P. (1986) Competition of Firms: Discriminatory Pricing and Location. Econometrica, 54, 623-640. http://dx.doi.org/10.2307/1911311 